\title{
FINANCIAL MARKETS WITH MEMORY I: DYNAMIC MODELS
}

\author{
V. ANH AND A. INOUE
}

\begin{abstract}
This is the first of two papers in which we consider a stock with price process defined by a stochastic differential equation driven by a process $Y(\cdot)$ different from Brownian motion. The adoption of such a colored noise input is motivated by an analysis of real market data. The process $Y(\cdot)$ is defined by a continuous-time $\operatorname{AR}(\infty)$-type equation and may have either short or long memory. We show that the process $Y(\cdot)$ has a good MA( $\infty)$-type representation. The existence of such simultaneous good $\mathrm{AR}(\infty)$ and $\mathrm{MA}(\infty)$ representations enables us to apply a new method for the calculation of relevant conditional expectations, whence to obtain various explicit results for problems such as portfolio optimization. The financial market defined by the above stock price process is complete, and if the coefficients are constant, then the prices of European calls and puts are given by the Black-Scholes formulas as in the Black-Scholes model. Unlike the latter, however, the model allows for differences between the historical and implied volatilities. The model includes a special case in which only two additional parameters are introduced to describe the memory of the market, compared with the Black-Scholes model. Analysis based on real market data shows that this simple model with two additional parameters is more realistic in capturing the memory effect of the market, while retaining the simplicity and usefulness of the Black-Scholes model.
\end{abstract}

\section{INTRODUCTION}

We consider a stock with price $S(t)$ at time $t \in[0, T]$. We suppose that $S(0)$ is a positive constant and that $S(\cdot)$ satisfies the stochastic differential equation

$$
d S(t)=S(t)[m(t) d t+\sigma(t) d Y(t)],
$$

where the process $m(\cdot)$ of mean rate of return and the volatility process $\sigma(\cdot)$ are progressively measurable and satisfy suitable integrability conditions. We also assume that $\sigma(t)>0$ for almost every $t \in[0, T]$ almost surely. For the filtration $\{\mathcal{F}(t)\}_{0 \leq t \leq T}$, we take the augmentation of the filtration generated by the process $(Y(t))_{0 \leq t \leq T}$.

1991 Mathematics Subject Classification. Primary 91B28; secondary 60G10, 60G20.

This work is partially supported by the Australian Research Council grant A10024117. 
In the standard model of financial markets (cf. [13, Chapter 1]), the process $Y(\cdot)$ is assumed to be a one-dimensional Brownian motion. In this paper, however, we assume that the process $Y(\cdot)$ is a continuous process with stationary increments such that $Y(0)=0$, and satisfies one of the following continuous-time $\operatorname{AR}(\infty)$-type equations:

$$
\frac{d Y}{d t}(t)+\int_{-\infty}^{t} a(t-s) \frac{d Y}{d t}(s) d s=\frac{d W}{d t}(t)
$$

or

$$
\frac{d Y}{d t}(t)-\int_{-\infty}^{t} a(t-s) \frac{d Y}{d t}(s) d s=\frac{d W}{d t}(t)
$$

(see (2.17) and (2.10) below for their precise formulation), where $(W(t))_{t \in \mathbf{R}}$ is a one-dimensional standard Brownian motion such that $W(0)=0$, and $d Y / d t$ and $d W / d t$ are the derivatives of $Y(\cdot)$ and $W(\cdot)$ respectively in the random distribution sense. The kernel $a(\cdot)$ is a nonnegative decreasing function with some adequate conditions to be specified below. The simplest case $a(\cdot)=0$ yields the usual white noise, i.e., $Y(\cdot)=W(\cdot)$, as the driving noise.

We need $Y(t)$ to be defined for $t \in \mathbf{R}$ to construct the process $Y(\cdot)$ but once it is constructed, we may regard $Y(t)$ as being defined for $t \in$ $[0, T]$. The integral on the left-hand side of (1.2) or (1.3) has the effect of incorporating memory into the dynamics of the process $Y(\cdot)$. The introduction of such process $Y(\cdot)$ as the driving force is motivated by an analysis based on real market data to be explained below.

In analogy with time series analysis, it is natural to introduce processes with memory by considering AR-type equations of the form (1.2) or (1.3). However, because of technical difficulties in continuous time, it is important to assume reasonable conditions. We assume that the delay kernel $a(\cdot)$ is a bounded, integrable, completely monotone function on $(0, \infty)$ satisfying some additional conditions. This assumption is essential and useful in our arguments. In fact, under the assumption, we develop a theory comparable to the discrete-time case, and in particular we show that $Y(\cdot)$ has a good $\mathrm{MA}(\infty)$-type representation

$$
Y(t)=W(t)-\int_{0}^{t}\left(\int_{-\infty}^{s} c(s-u) d W(u)\right) d s
$$

for $(1.2)$ or

$$
Y(t)=W(t)+\int_{0}^{t}\left(\int_{-\infty}^{s} c(s-u) d W(u)\right) d s
$$

for (1.3) (Theorems 2.7, 2.11 and 2.13). We find that the kernel $c(\cdot)$ is also completely monotone, a fact which is helpful in the analysis of $Y(\cdot)$, whence that of $S(\cdot)$. Using (1.4) or (1.5), we show that $Y(\cdot)$ is a Gaussian 
semimartingale with respect to the filtration $\{\mathcal{F}(t)\}_{0 \leq t \leq T}$ stated above (Theorem 3.1). In particular, we can define the SDE (1.1). The explicit form of the semimartingale representation will be derived in [3]. It should be noticed that (1.4) or (1.5) is not a semimartingale representation of $Y(\cdot)$ since the Brownian motion $W(\cdot)$ is not $\{\mathcal{F}(t)\}$-adapted.

We consider a financial market consisting of a stock with the above price process $(S(t))_{0 \leq t \leq T}$ and a share in the money market with price process $\left(S_{0}(t)\right)_{0 \leq t \leq T}$ defined by

$$
S_{0}(0)=1, \quad d S_{0}(t)=r(t) S_{0}(t) d t \quad(t \in[0, T]),
$$

where the interest rate process $r(\cdot)$ is progressively measurable and satisfies suitable integrability conditions. The process $Y(\cdot)$ may have short or long memory according to the choice of $a(\cdot)$. In either case, we show that the financial market is complete. Moreover, if $\sigma(\cdot)$ in $(1.1)$ and $r(\cdot)$ are constant, then the behavior of the discounted price process $\tilde{S}(t)=S(t) / S_{0}(t)$ under the equivalent martingale measure is equal to that in the BlackScholes (BS) environment, whence the prices of European calls and puts in the market are given by the BS formulas as in the BS model (Theorem 3.3). However, unlike the BS model, the above model allows for differences between the historical volatility $\mathrm{HV}(\cdot)$ and the volatility implied by the BS formulas (Theorems 4.2, 4.4 and 4.6). Here we define $\mathrm{HV}(\cdot)$ by

$$
\operatorname{HV}(t-s):=\sqrt{\frac{\operatorname{Var}\{\log (S(t) / S(s))\}}{t-s}} \quad(t>s \geq 0) .
$$

In order to allow for long memory (Beran [4], Anh and Heyde [1]) in the dynamics of a stock price process $S(\cdot)$, attempts have been made to replace Brownian motion by fractional Brownian motion $B_{H}(\cdot)$ with Hurst index $1 / 2<H<1$ (Lin [14], Cutland et al. [6], Comte and Renault [7, 8], Willinger et al. [18]). However this approach is not entirely satisfactory since fractional Brownian motion is not a semimartingale (Liptser and Shiryaev [15], Lin [14], Rogers [17]), and as a result, the market is not arbitrage free (Cutland et al. [6], Rogers [17]). Our process $S(\cdot)$ has the advantage that the corresponding market is complete and, if $\sigma(\cdot)$ is constant, then the same option pricing formulas as in the BS model hold.

As stated above, the stationary increment process $Y(\cdot)$ possesses simultaneously good $\mathrm{AR}(\infty)$ - and $\mathrm{MA}(\infty)$ - type representations (1.2) and (1.4), or (1.3) and (1.5). This fact turns out to be a great advantage of the model, as will be illustrated in our second paper [3] in which methods of calculating conditional expectations relevant to $Y(\cdot)$ are developed. This is done by applying a new method for prediction, in which both $\operatorname{AR}(\infty)$ - and $\mathrm{MA}(\infty)$ - type representations play an important role. In [3], the semimartingale representation of $Y(\cdot)$ is derived in explicit form by 
the method, and, by applying the representation, the expected log-utility maximization problem for the financial market model is solved completely.

The simplest case of our model is the one in which the coefficients $\sigma(\cdot)$ and $m(\cdot)$ are constant and the kernel $a(\cdot)$ in (1.2) or (1.3) is given by $a(t)=p e^{-q t}$ for $t>0$, where $p$ and $q$ are positive constants (Examples 4.3 and 4.5). For (1.3), we also assume $p<q$. This simplest model is worth special attention. This is a parametric model which has only two additional parameters $p$ and $q$ describing the memory of the market, compared with the BS model. Clearly, this simplicity is a significant advantage in parameter estimation. In [2], we estimated $\operatorname{HV}(t)(t=1,2,3, \ldots)$ from real market data, such as the closing values of S\&P 500 from November 2001 through May 2002. It is found that, unlike in the BS model, the estimated $\mathrm{HV}(\cdot)$ is not constant, and very often reveals features in agreement with those described by the model with two additional parameters. We also fitted $\mathrm{HV}(\cdot)$ of this simple model by using nonlinear least squares, and found that it approximates the estimated graph of $\mathrm{HV}(\cdot)$ very well for the $\mathrm{S} \& \mathrm{P} 500$ data. The introduction of the process $Y(\cdot)$ as the driving noise in (1.1) has been strongly motivated by this observation. The BS model is still dominant among many market models used by practitioners. This would be due to the simplicity of the BS model. The model with two additional parameters is realistic in capturing a new aspect which may be regarded as memory effect, while keeping the simplicity and usefulness of the BS model.

In Section 2, we define and study the process $Y(\cdot)$. In Sections 3 and 4, we apply the results in Section 2 to study the financial market model. In Section 5, we prove two theorems which we need in Section 2.

\section{THE DRIVING NOISE}

In this section, we consider the process $Y(\cdot)$ which drives the SDE (1.1). The dynamics of $Y(\cdot)$ is given by the continuous-time $\operatorname{AR}(\infty)$-type equation (1.2) or (1.3).

We start from the correspondences between two measures $\mu$ and $\nu$ on $(0, \infty)$ through the relation

$$
\left\{1+\int_{0}^{\infty} \frac{1}{s-i z} \nu(d s)\right\}\left\{1-\int_{0}^{\infty} \frac{1}{s-i z} \mu(d s)\right\}=1 \quad(\Im z>0) .
$$

We need such results to study correspondences between the kernels $a(\cdot)$ and $c(\cdot)$ in (1.2) and (1.4) or (1.3) and (1.5). We define

$$
\mathcal{M}_{0}=\left\{\mu: \begin{array}{l}
\mu \text { is a (possibly zero) Borel measure on }(0, \infty) \\
\text { such that } \int_{0}^{\infty} s^{-1} \mu(d s)<1
\end{array}\right\},
$$




$$
\mathcal{N}_{0}=\left\{\nu: \begin{array}{l}
\nu \text { is a (possibly zero) Borel measure on }(0, \infty) \\
\text { such that } \int_{0}^{\infty} s^{-1} \nu(d s)<\infty
\end{array}\right\}
$$

Theorem 2.1. For $\mu \in \mathcal{M}_{0}$, there exists a unique $\nu \in \mathcal{N}_{0}$ satisfying (2.1). Conversely, for $\nu \in \mathcal{N}_{0}$, there exists a unique $\mu \in \mathcal{M}_{0}$ satisfying (2.1).

We can prove Theorem 2.1, as well as Theorem 2.3 below, by the standard arguments involving approximation by discrete measures and the Helly selection principle. We give their proofs in Section 5.

Example 2.2. Let $\mu=p \delta_{q}$ with $0<p<q$. Then $\int_{0}^{\infty} s^{-1} \mu(d s)<1$, and so $\mu \in \mathcal{M}_{0}$. The measure $\nu$ that corresponds to $\mu$ by Theorem 2.1 is given by $p \delta_{q-p}$ since

$$
\left\{1-\int_{0}^{\infty} \frac{1}{s-i z} \mu(d s)\right\}^{-1}-1=\frac{p}{q-p-i z} .
$$

We next define

$$
\begin{aligned}
& \mathcal{M}_{1}=\left\{\mu: \begin{array}{l}
\mu \text { is a Borel measure on }(0, \infty) \text { such that } \\
\int_{0}^{\infty} s^{-1} \mu(d s)=1, \int_{0}^{\infty} s^{-2} \mu(d s)=\infty
\end{array}\right\}, \\
& \mathcal{N}_{1}=\left\{\nu: \begin{array}{l}
\nu \text { is a Borel measure on }(0, \infty) \text { such that } \\
\int_{0}^{\infty}(s+1)^{-1} \nu(d s)<\infty, \int_{0}^{\infty} s^{-1} \nu(d s)=\infty
\end{array}\right\} .
\end{aligned}
$$

Theorem 2.3. For $\mu \in \mathcal{M}_{1}$, there exists a unique $\nu \in \mathcal{N}_{1}$ satisfying (2.1). Conversely, for $\nu \in \mathcal{N}_{1}$, there exists a unique $\mu \in \mathcal{M}_{1}$ satisfying (2.1).

Definition 2.4. We define the one-to-one and onto maps $\mathcal{M}_{0} \ni \mu \mapsto$ $\theta_{0}(\mu)=\nu \in \mathcal{N}_{0}$ and $\mathcal{M}_{1} \ni \mu \mapsto \theta_{1}(\mu)=\nu \in \mathcal{N}_{1}$ by $(2.1)$.

Lemma 2.5. For $\mu \in \mathcal{M}_{0}$ (resp. $\mu \in \mathcal{M}_{1}$ ), we set $\nu:=\theta_{0}(\mu) \in \mathcal{N}_{0}$ (resp. $\left.\nu:=\theta_{1}(\mu) \in \mathcal{N}_{1}\right)$. Then $\mu(0, \infty)=\nu(0, \infty)$. In particular, $\mu(0, \infty)<\infty$ if and only if $\nu(0, \infty)<\infty$.

Proof. We have

$$
\nu(0, \infty)=\lim _{y \uparrow \infty} \int_{0}^{\infty} \frac{y}{y+s} \nu(d s)=\lim _{y \uparrow \infty} \frac{\int_{0}^{\infty} y /(y+s) \mu(d s)}{1-\int_{0}^{\infty} 1 /(y+s) \mu(d s)}=\mu(0, \infty),
$$

which yields the lemma.

We write $H$ for the complex Hilbert space

$$
H=\left\{a \in L^{2}(\Omega, \mathcal{F}, P): E[a]=0\right\}
$$

with inner product $(a, b)_{H}=E[a \bar{b}]$ and norm $\|a\|_{H}=(a, a)_{H}^{1 / 2}$. Let $\mathcal{D}(\mathbf{R})$ be the space of all $\phi \in C^{\infty}(\mathbf{R})$ with compact support, endowed with the usual topology. A random distribution $X$ (with expectation zero) is a 
linear continuous map from $\mathcal{D}(\mathbf{R})$ to $H$. We write $D X$ for its derivative. For $t \in \mathbf{R}$, we write $M(X)$ (resp. $M_{t}(X)$ ) for the closed linear hull of $\{X(\phi): \phi \in \mathcal{D}(\mathbf{R})\}$ (resp. $\{X(\phi): \phi \in \mathcal{D}(\mathbf{R})$, supp $\phi \subset(-\infty, t]\})$ in $H$. For $k \in L^{1}(\mathbf{R}, d t)$ and a stationary random distribution $X$, we define the convolution $k * X$, which is also a stationary random distribution, by

$$
(k * X)(\phi):=\int_{-\infty}^{\infty} k(u) X\left(\tau_{u} \phi\right) d u \quad(\phi \in \mathcal{D}(\mathbf{R}))
$$

where $\tau_{u} \phi(t):=\phi(t+u)$ and the integral on the right-hand side is an $H$-valued Bochner integral. We refer to [10, Section 2] for details.

For a Borel measure $\nu$ on $(0, \infty)$ such that $\int_{0}^{\infty}(s+1)^{-1} \nu(d s)<\infty$, we write

$$
k_{\nu}(t):=I_{(0, \infty)}(t) \int_{0}^{\infty} e^{-t s} \nu(d s) \quad(t \in \mathbf{R}) .
$$

Let $(W(t))_{t \in \mathbf{R}}$ be a one-dimensional standard Brownian motion such that $W(0)=0$, defined on a probability space $(\Omega, \mathcal{F}, P)$. Since $W(\cdot)$ is a process with stationary increments, the derivative $D W$ is a stationary random distribution (see [11]).

First we consider the following equation which corresponds to (1.3) in the long-memory case:

$$
X-a * X=D W,
$$

where $a(\cdot)$ is a function satisfying the following condition:

(L) $\left\{\begin{array}{l}a(t)=k_{\mu}(t) \text { for } t \in \mathbf{R} \text {, where } \mu \text { is a finite Borel measure on } \\ (0, \infty) \text { satisfying } \int_{0}^{\infty} s^{-1} \mu(d s)=1, \int_{0}^{\infty} s^{-2} \mu(d s)=\infty \text {, and (L1) }\end{array}\right.$

with the condition (L1) being given below. Note that (2.3) can be written formally as

$$
X(t)-\int_{-\infty}^{t} a(t-s) X(s) d s=\frac{d W}{d t}(t)
$$

For a Borel measure $\nu$ on $(0, \infty)$ satisfying

$$
\int_{0}^{\infty} k_{\nu}(t)^{2} d t<\infty
$$

we define a real, centered, stationary Gaussian process $(U(t))_{t \in \mathbf{R}}$ by

$$
U(t):=\int_{-\infty}^{t} k_{\nu}(t-s) d W(s) \quad(t \in \mathbf{R}) .
$$

Then $U(\cdot)$ is purely nondeterministic, and (2.6) corresponds to the socalled canonical representation of $U(\cdot)$; thus, $M_{t}\left(U_{\nu}\right)=M_{t}(D W)$ for 
$t \in \mathbf{R}$. The spectral representation of $U(\cdot)$, as a stationary random distribution, is given by

$$
U(\phi)=\int_{-\infty}^{\infty} F_{\nu}(\xi) \hat{\phi}(\xi) Z_{D W}(d \xi) \quad(\phi \in \mathcal{D}(\mathbf{R})),
$$

where $Z_{D W}$ is the random measure associated with $D W$ satisfying

$$
\left(Z_{D W}(A), Z_{D W}(B)\right)_{H}=\frac{1}{2 \pi} \int_{A \cap B} d \xi,
$$

$\hat{\phi}$ is the Fourier transform of $\phi$ defined by $\hat{\phi}(\xi):=\int_{-\infty}^{\infty} e^{-i t \xi} \phi(\xi) d \xi$, and $F_{\nu}(\cdot)$ is a function defined by

$$
F_{\nu}(z):=\int_{0}^{\infty} \frac{1}{s-i z} \nu(d s) \quad(\Im z \geq 0) .
$$

We refer to [10] for these results. We write $\gamma(\cdot)$ for the autocovariance function of $U(\cdot)$ :

$$
\gamma(t):=E[U(t) U(0)] \quad(t \in \mathbf{R}) .
$$

Then we have $\gamma(t)=\int_{0}^{\infty} k_{\nu}(|t|+s) k_{\nu}(s) d s$.

If $\nu$ is a finite measure in $\mathcal{N}_{1}$ such that

$$
\int_{1}^{\infty} k_{\nu}(t)^{2} d t<\infty
$$

then $\nu$ satisfies $(2.5)$ since

$$
\int_{0}^{1} k_{\nu}(t)^{2} d t \leq k_{\nu}(0+) \int_{0}^{1} k_{\nu}(t) d t<\infty .
$$

In this case, from $\int_{0}^{\infty} k_{\nu}(t) d t=\infty$, we see that the stationary Gaussian process $U(\cdot)$ defined by $(2.6)$ is long-memory in the sense that $\int_{0}^{\infty} \gamma(t) d t=$ $\infty$ (cf. [10]). Now we define the condition (L1) above for $\mu \in \mathcal{M}_{1}$ by

$$
\nu=\theta_{1}(\mu) \text { satisfies }(2.8) \text {. }
$$

We write $M(D W)^{\perp}$ for the orthogonal complement to $M(D W)$ in $H$. In the next theorem, we solve the equation (2.3) explicitly. Notice that if $\mu$ satisfies (L), then $\mu \in \mathcal{M}_{1}$, whence we can consider $\theta_{1}(\mu)$.

Theorem 2.6. Let $a(\cdot)$ be a function satisfying $(\mathrm{L})$, and let $\nu:=\theta_{1}(\mu)$. Then a stationary random distribution $X$ satisfies $(2.3)$ if and only if $X=X_{0}+b$, where $b \in M(D W)^{\perp}$ and $X_{0}$ is the stationary random distribution defined by

$$
X_{0}=U+D W
$$

with $U(\cdot)$ in (2.6). In particular, $X_{0}$ is the only purely nondeterministic stationary random distribution that satisfies (2.3). 
Proof. The arguments below are similar to those of the proofs of [10, Theorems 3.4 and 3.8]. Let $X$ be a stationary random distribution satisfying (2.3). As in [10, Proposition 2.3], we have

$$
(X-a * X)(\phi)=\int_{-\infty}^{\infty}\left\{1-F_{\mu}(\xi)\right\} \hat{\phi}(\xi) Z_{X}(d \xi)
$$

where $Z_{X}$ is the random measure associated with the spectral representation of $X$. From this, we see that $X$ and $D W=X-a * X$ are stationarily correlated (cf. [10, Section 2]). We define a random distribution $P_{D W} X$ by $P_{D W} X(\phi)=p_{D W}(X(\phi))$, where $p_{D W}$ is the orthogonal projection operator from $H$ onto $M(D W)$. We set $X_{1}:=X-P_{D W} X$. Then, by [10, Theorem 2.1], $X_{1}$ is a stationary random distribution satisfying $X_{1}=a * X_{1}$. We write $\mu_{X_{1}}$ for the spectral measure of $X_{1}$. Then we have

$$
\left\|\left(X_{1}-a * X_{1}\right)(\phi)\right\|_{H}^{2}=\int_{-\infty}^{\infty}|\hat{\phi}(\xi)|^{2}\left|1-F_{\mu}(\xi)\right|^{2} \mu_{X_{1}}(d \xi) .
$$

Since it holds that, for $\xi \neq 0$,

$$
\Re\left\{1-F_{\mu}(\xi)\right\}=1-\int_{0}^{\infty} \frac{s}{s^{2}+\xi^{2}} \mu(d s)>1-\int_{0}^{\infty} s^{-1} \mu(d s)=0,
$$

we have

$$
\left|1-F_{\mu}(\xi)\right|^{2}>0 \quad(\xi \neq 0), \quad=0 \quad(\xi=0),
$$

whence $X-P_{D W} X=X_{1}=b$ for some $b \in M(D W)^{\perp}$.

We set $X_{0}:=P_{D W} X$. Then, again by [10, Theorem 2.1], there exists $g \in L^{2}\left(\mathbf{R},\left(1+x^{2}\right)^{-k} d \xi\right)$, for some $k \in \mathbf{N} \cup\{0\}$, such that

$$
X_{0}(\phi)=\int_{-\infty}^{\infty} g(\xi) \hat{\phi}(\xi) Z_{D W}(d \xi)
$$

The spectral measure $\nu_{D W}$ of $D W$ is given by $\mu_{D W}(d \xi)=(2 \pi)^{-1} d \xi$. Hence from

$$
a * X_{0}(\phi)=\int_{-\infty}^{\infty} F_{\mu}(\xi) g(\xi) \hat{\phi}(\xi) Z_{D W}(d \xi),
$$

it follows that, for $\phi \in \mathcal{D}(\mathbf{R})$,

$$
\begin{aligned}
0 & =\left\|X_{0}(\phi)-a * X_{0}(\phi)-D W(\phi)\right\|_{H}^{2} \\
& =\frac{1}{2 \pi} \int_{-\infty}^{\infty}|\hat{\phi}(\xi)|^{2}\left|\left\{\left(1-F_{\mu}(\xi)\right) g(\xi)-1\right\}\right|^{2} d \xi
\end{aligned}
$$

This implies

$$
g(\xi)=\frac{1}{1-F_{\mu}(\xi)}=F_{\nu}(\xi)+1
$$

hence

$$
X_{0}(\phi)=\int_{-\infty}^{\infty}\left\{F_{\nu}(\xi)+1\right\} \hat{\phi}(\xi) Z_{D W}(d \xi)=U(\phi)+D W(\phi)
$$


Thus $X_{0}$ is given by (2.9). Conversely, we can easily show that $X=X_{0}+b$ with $(2.9)$ and $b \in M(D W)^{\perp}$ is a stationary random distribution that satisfies (2.3).

For $z=x+i y$ with $y>0$, it holds that

$$
\Re\left\{F_{\nu}(z)+1\right\}=1+\int_{0}^{\infty} \frac{s+y}{(s+y)^{2}+x^{2}} \nu(d s)>0 .
$$

This implies that $f(z):=F_{\nu}(z)+1$ is an outer function on the upper half-plane $\Im z>0$. Thus

$$
f(z):=\exp \left\{\frac{1}{\pi i} \int_{-\infty}^{\infty} \frac{1+t z}{t-z} \cdot \frac{\log |f(t)|}{1+t^{2}} d t\right\} \quad(\Im z>0) .
$$

It follows that

$$
M_{t}\left(X_{0}\right)=M_{t}(D W) \quad(t \in \mathbf{R})
$$

(cf. [9]), so that $M_{t}\left(X_{0}+b\right)=M_{t}(D W)+\mathbf{C} b$ for $b \in M(D W)^{\perp}$ or

$$
\bigcap_{t} M_{t}\left(X_{0}+b\right)=\left\{\bigcap_{t} M_{t}\left(X_{0}\right)\right\} \oplus \mathbf{C} b=\left\{\bigcap_{t} M_{t}(D W)\right\} \oplus \mathbf{C} b=\mathbf{C} b .
$$

Therefore $X_{0}+b$ with $b \in M(D W)^{\perp}$ is purely nondeterministic if and only if $b=0$.

We are now ready to consider the equation (1.3) in the long-memory case.

Theorem 2.7. Let $a(\cdot)$ and $\nu$ be as in Theorem 2.6. Let $(Y(t))_{t \in \mathbf{R}}$ be a zero-mean, mean-square continuous process with stationary increments such that $Y(0)=0$. We assume that DY is purely nondeterministic and that it satisfies

$$
D Y-a * D Y=D W .
$$

Then the process $Y(\cdot)$ is the Gaussian process given by (1.5) for $t \in \mathbf{R}$ with $c(\cdot)=k_{\nu}(\cdot)$. The kernel $c(\cdot)$ is determined from $a(\cdot)$ through the relation

$$
\left\{1-\int_{0}^{\infty} e^{i z t} a(t) d t\right\}\left\{1+\int_{0}^{\infty} e^{i z t} c(t) d t\right\}=1 \quad(\Im z>0) .
$$

Theorem 2.7 follows immediately from Theorem 2.6 and [11, Theorem 6.1]. It should be noticed that (2.10) can be written formally as (1.3).

We give a sufficient condition for (L1) in terms of the asymptotic behavior of $a(t)$ as $t \rightarrow \infty$.

Lemma 2.8. Let $0<p<1$ and let $\ell(\cdot)$ be a slowly varying function at infinity. Let $\mu \in \mathcal{M}_{1}$ and define $\nu \in \mathcal{N}_{1}$ by $\nu:=\theta_{1}(\mu)$. We set $a(\cdot):=k_{\mu}(\cdot)$ and $c(\cdot)=k_{\nu}(\cdot)$. Then

$$
a(t) \sim t^{-(p+1)} \ell(t) p \quad(t \rightarrow \infty)
$$


if and only if

$$
c(t) \sim \frac{t^{-(1-p)}}{\ell(t)} \cdot \frac{\sin (p \pi)}{\pi} \quad(t \rightarrow \infty)
$$

Proof. We prove only $(2.12) \Rightarrow(2.13)$; the converse implication $(2.13) \Rightarrow$ (2.12) can be proved in the same way. Since $\int_{0}^{\infty} k_{\mu}(t) d t=1$, we have, by integration by parts,

$$
1-\int_{0}^{\infty} e^{-t y} k_{\mu}(t) d t=y \int_{0}^{\infty} e^{-t y}\left(\int_{t}^{\infty} k_{\mu}(s) d s\right) d t \quad(y>0) .
$$

Hence

$$
\int_{0}^{\infty} e^{-t y} k_{\nu}(t) d t=\frac{\int_{0}^{\infty} e^{-t y} k_{\mu}(t) d t}{1-\int_{0}^{\infty} e^{-t y} k_{\mu}(t) d t}=\frac{\int_{0}^{\infty} e^{-t y} k_{\mu}(t) d t}{y \int_{0}^{\infty} e^{-t y}\left(\int_{t}^{\infty} k_{\mu}(s) d s\right) d t}
$$

Now (2.12) implies $\int_{t}^{\infty} k_{\mu}(s) d s \sim t^{-p} \ell(t)$ as $t \rightarrow \infty$, so that

$$
y \int_{0}^{\infty} e^{-t y}\left(\int_{t}^{\infty} k_{\mu}(s) d s\right) d t \sim y^{p} \ell(1 / y) \Gamma(1-p) \quad(y \downarrow 0)
$$

(cf. [5, Theorem 1.7.6]). On the other hand, $\lim _{y \downarrow 0} \int_{0}^{\infty} e^{-t y} k_{\mu}(t) d t=1$. Thus

$$
y \int_{0}^{\infty} e^{-t y} k_{\nu}(t) d t \sim \frac{y^{1-p}}{\ell(1 / y) \Gamma(1-p)} \quad(y \downarrow 0) .
$$

By Karamata's Tauberian theorem (cf. [5, Theorem 1.7.6]), this implies (2.13).

Theorem 2.9. Let $0<p<1 / 2$ and let $\ell(\cdot)$ be a slowly varying function at infinity. Let $\mu$ be a finite measure in $\mathcal{M}_{1}$. We put $a(\cdot):=k_{\mu}(\cdot)$ and assume (2.12). Then $\mu$ satisfies (L1), whence $a(\cdot)$ satisfies $(\mathrm{L})$. If we put $\nu:=\theta_{1}(\mu)$ and $c(\cdot)=k_{\nu}(\cdot)$, then $k_{\nu}(\cdot)$ satisfies (2.13), and the autocovariance function $\gamma(\cdot)$ of the stationary process $U(t)=\int_{-\infty}^{t} c(t-s) d W(s)$ satisfies

$$
\gamma(t) \sim \frac{t^{-(1-2 p)}}{\ell(t)^{2}}\left(\frac{\sin (p \pi)}{\pi}\right)^{2} B(1-2 p, p) \quad(t \rightarrow \infty) .
$$

Proof. By Lemma $2.8, k_{\nu}(\cdot)$ satisfies $(2.13)$, so that $(2.8)$ holds. Thus $\mu$ satisfies (L1), whence $a(\cdot)$ satisfies (L). By [10, Proposition 4.3] and the equality $\gamma(t)=\int_{0}^{\infty} c(t+s) c(s) d s,(2.3)$ implies (2.14).

Example 2.10. For $0<p<1 / 2$, set $\mu(d s):=\Gamma(p)^{-1} s^{p} e^{-s} d s$ and $a(\cdot)=$ $k_{\mu}(\cdot)$. Then we have

$$
a(t)=\frac{p}{(t+1)^{p+1}} \quad(t>0)
$$


Since $a(0+)<\infty, \int_{0}^{\infty} a(t) d t=1, \int_{0}^{\infty} d t \int_{t}^{\infty} a(s) d s=\infty$, and $a(t) \sim$ $p t^{-(p+1)}$ as $t \rightarrow \infty$, we find that $a(\cdot)$ satisfies the condition of Theorem 2.7, that is, $(\mathrm{L})$; take $\ell(\cdot)=1$ in Theorem 2.9 .

Next we consider the equation (1.3) or (2.10) in the short-memory case. We assume that $a(\cdot)$ satisfies the following condition $(\mathrm{S} 1)$ :

$$
\left\{\begin{array}{l}
a(t)=k_{\mu}(t) \text { for } t \in \mathbf{R}, \text { where } \mu \text { is a (possibly zero) finite Borel } \\
\text { measure on }(0, \infty) \text { such that } \int_{0}^{\infty} s^{-1} \mu(d s)<1
\end{array}\right.
$$

If $\nu$ is a finite measure in $\mathcal{N}_{0}$, then

$$
\int_{0}^{\infty} k_{\nu}(t)^{2} d t \leq k_{\nu}(0+) \int_{0}^{\infty} k_{\nu}(t) d t<\infty .
$$

Thus $\nu$ satisfies (2.5), whence we may define a stationary process $U(\cdot)$ by (2.6). In this case, from $\int_{0}^{\infty} k_{\nu}(t) d t<\infty$, we see that $U(\cdot)$ is short-memory in the sense that its autocovariance function $\gamma(\cdot)$ satisfies $\int_{0}^{\infty} \gamma(t) d t<\infty$.

Theorem 2.11. Let $a(\cdot)$ be a function satisfying $(\mathrm{S} 1)$, and let $(Y(t))_{t \in \mathbf{R}}$ be a zero-mean, mean-square continuous process with stationary increments such that $Y(0)=0$. We assume that DY satisfies (2.10). Then the process $Y(\cdot)$ is the Gaussian process given by (1.5) for $t \in \mathbf{R}$ with $c(\cdot)=k_{\nu}(\cdot)$, where $\nu:=\theta_{0}(\mu)$. The kernel $c(\cdot)$ is determined from $a(\cdot)$ through (2.11). The stationary random distribution DY is purely nondeterministic.

The proof of Theorem 2.11 is similar to that of Theorem 2.7; and so we omit it. Notice that, in Theorem 2.11, unlike in Theorem 2.7, we need not a priori assume that $D Y$ is purely nondeterministic.

Example 2.12. Let $0<p<q$ and $\mu=p \delta_{q}$ as in Example 2.2. We put $a(\cdot)=k_{\mu}(\cdot)$. Then $a(\cdot)$ satisfies (S1). In this case, we have $a(t)=p e^{-q t}$ and $c(t)=p e^{-(q-p) t}$ for $t>0$. Thus the equation (1.3) may be written as

$$
\frac{d Y}{d t}(t)-\int_{-\infty}^{t} p e^{-q(t-s)} \frac{d Y}{d t}(s) d s=\frac{d W}{d t}(t),
$$

and the solution $Y(\cdot)$ is given by

$$
Y(t)=W(t)+\int_{0}^{t}\left(\int_{-\infty}^{s} p e^{-(q-p)(s-u)} d W(u)\right) d s \quad(t \in \mathbf{R}) .
$$

Let $W(\cdot)$ be as above. Finally we consider the equation

$$
D Y+a * D Y=\sigma D W
$$


which can be formally written as (1.2). We assume that the kernel $a(\cdot)$ satisfies the following condition (S2):

$$
\left\{\begin{array}{l}
a(t)=k_{\nu}(t) \text { for } t \in \mathbf{R}, \text { where } \nu \text { is a (possibly zero) finite Borel } \\
\text { measure on }(0, \infty) \text { such that } \int_{0}^{\infty} s^{-1} \nu(d s)<\infty
\end{array}\right.
$$

For $\mu:=\theta_{0}^{-1}(\nu) \in \mathcal{M}_{0}$, it follows from Lemma 2.5 that $\mu(0, \infty)<\infty$. Hence we see that $\int_{0}^{\infty} k_{\mu}(t)^{2} d t<\infty$. So, in the same way as above, we may define a real centered stationary Gaussian process $U(\cdot)$ by

$$
U(t):=\int_{-\infty}^{t} k_{\mu}(t-s) d W(s) \quad(t \in \mathbf{R}) .
$$

Theorem 2.13. Let $a(\cdot)$ be a function satisfying $(\mathrm{S} 2)$, and let $(Y(t))_{t \in \mathbf{R}}$ be a zero-mean, mean-square continuous process with stationary increments such that $Y(0)=0$. We assume that DY satisfies (2.17). Then the process $Y(\cdot)$ is the Gaussian process given by (1.4) for $t \in \mathbf{R}$ with $c(\cdot)=k_{\mu}(\cdot)$, where $\mu:=\theta_{0}^{-1}(\nu)$. The kernel $c(\cdot)$ is determined from $a(\cdot)$ through the relation

$$
\left\{1+\int_{0}^{\infty} e^{i z t} a(t) d t\right\}\left\{1-\int_{0}^{\infty} e^{i z t} c(t) d t\right\}=1 \quad(\Im z>0) .
$$

The stationary random distribution DY is purely nondeterministic.

The proof of Theorem 2.13 is similar to that of Theorem 2.7; and so we omit it.

Example 2.14. For $p, q \in(0, \infty)$, we set $\nu=p \delta_{q}$ and $a(\cdot)=k_{\nu}(\cdot)$. Then we have $a(t)=p e^{-q t}$ for $t>0$. Since

$$
\left(1+\frac{p}{q-i z}\right)\left(1-\frac{p}{p+q-i z}\right)=1
$$

the measure $\mu:=\theta_{0}^{-1}(\nu)$ is given by $p \delta_{p+q}$, so that $c(t)=p e^{-(p+q) t}$ for $t>0$. Thus the equation (1.2) may be written as

$$
\frac{d Y}{d t}(t)+\int_{-\infty}^{t} p e^{-q(t-s)} \frac{d Y}{d t}(s) d s=\frac{d W}{d t}(t),
$$

and the solution $Y(\cdot)$ is given by

$$
Y(t)=W(t)-\int_{0}^{t}\left(\int_{-\infty}^{s} p e^{-(q-p)(s-u)} d W(u)\right) d s \quad(t \in \mathbf{R}) .
$$




\section{THE FINANCIAL MARKET}

In this section, we consider a financial market consisting of a stock with price $S(t)$ defined by (1.1) as well as a share in the money market.

In this section, we assume that the probability space $(\Omega, \mathcal{F}, P)$ is complete. Let $W(\cdot), Y(\cdot)$ and $c(\cdot)$ be as in Theorem 2.7 or 2.11 or 2.13 . We put

$$
\epsilon:= \begin{cases}+1 & \text { for } Y(\cdot) \text { with }(2.17) \text { whence }(1.4) \\ -1 & \text { for } Y(\cdot) \text { with }(2.10) \text { whence }(1.5)\end{cases}
$$

As in Section 2, we define a continuous stationary Gaussian process $U(\cdot)$ by

$$
U(t)=\int_{-\infty}^{t} c(t-s) d W(s) \quad(t \in \mathbf{R}) .
$$

Let $T$ be a positive constant. We define the filtration $\{\mathcal{F}(t)\}_{0 \leq t \leq T}$ by

$$
\left\{\begin{array}{l}
\{\mathcal{F}(t)\}_{0 \leq t \leq T} \text { is the augmentation, by the null sets in } \mathcal{F}^{Y}(T), \\
\text { of the filtration }\left\{\mathcal{F}^{Y}(t)\right\}_{0 \leq t \leq T},
\end{array}\right.
$$

where $\left\{\mathcal{F}^{Y}(t)\right\}_{0 \leq t \leq T}$ is the filtration generated by $Y(\cdot)$, i.e.,

$$
\mathcal{F}^{Y}(t):=\sigma(Y(u): 0 \leq u \leq t) \quad(0 \leq t \leq T) .
$$

We define another Gaussian process $(\alpha(t))_{0 \leq t \leq T}$ by

$$
\alpha(t)=E[U(t) \mid \mathcal{F}(t)] \quad(0 \leq t \leq T) .
$$

Following the Kailath-Shiryaev construction of innovations, we also define a process $(B(t))_{0 \leq t \leq T}$ by

$$
B(t)=Y(t)+\epsilon \int_{0}^{t} \alpha(s) d s \quad(0 \leq t \leq T) .
$$

The next theorem follows directly from [16, Theorem 7.16].

Theorem 3.1. Under $P, B(\cdot)$ is a Brownian motion such that the filtration $\{\mathcal{F}(t)\}_{0 \leq t \leq T}$ is equal to the augmentation, by the null sets in $\mathcal{F}^{B}(T)$, of the filtration $\left\{\mathcal{F}^{B}(t)\right\}_{0 \leq t \leq T}$ generated by $B(\cdot)$. In particular, $(Y(t))_{0 \leq t \leq T}$ is a Gaussian semimartingale with respect to $\{\mathcal{F}(t)\}_{0 \leq t \leq T}$, and (3.5) gives its semimartingale representation.

We assume the following conditions:

(i) $r(\cdot)$ is a progressively measurable process satisfying $\int_{0}^{T}|r(t)| d t<\infty$ a.s.;

(ii) $m(\cdot)$ is a progressively measurable process satisfying $\int_{0}^{T}|m(t)| d t<\infty$ a.s.;

(iii) $\sigma(\cdot)$ is a progressively measurable process satisfying $\int_{0}^{T} \sigma^{2}(t) d t<\infty$ a.s. and $\sigma(t)>0$ Lebesgue-almost-every $t \in[0, t]$ a.s. 
A share of the money market has price $S_{0}(t)$ at time $t \in[0, T]$ with (1.6), or equivalently,

$$
S_{0}(t)=\exp \left\{\int_{0}^{t} r(u) d u\right\} \quad(t \in[0, T]) .
$$

A stock has price $S(t)$ at time $t \in[0, T]$, with $S(0)$ a positive constant, satisfying the equation (1.1). By Theorem 3.1, (1.1) can be written as

$$
d S(t)=S(t)\{[m(t)-\epsilon \sigma(t) \alpha(t)] d t+\sigma(t) d B(t)\} .
$$

Thus the solution of (1.1) is given by, for $0 \leq t \leq T$,

$$
\begin{aligned}
S(t)= & S(0) \exp \left\{\int_{0}^{t} \sigma(s) d B(s)\right. \\
& \left.\quad+\int_{0}^{t}\left[m(s)-\epsilon \sigma(s) \alpha(s)-\frac{1}{2} \sigma^{2}(s)\right] d s\right\} \\
= & S(0) \exp \left\{\int_{0}^{t} \sigma(s) d Y(s)+\int_{0}^{t}\left[m(s)-\frac{1}{2} \sigma^{2}(s)\right] d s\right\} .
\end{aligned}
$$

We consider the financial market

$$
\mathcal{M}=\left\{(\Omega, \mathcal{F}, P),\{\mathcal{F}(t)\}_{0 \leq t \leq T},(S(t))_{0 \leq t \leq T},\left(S_{0}(t)\right)_{0 \leq t \leq T}\right\} .
$$

By Theorem 3.1, we see that $\mathcal{M}$ may be regarded as a financial market in the sense of [13, Definition 1.1.3]. In what follows, we use the definitions of [13] such as completeness of a market. We consider the following conditions for $\mathcal{M}$ :

$$
\int_{0}^{T} \theta^{2}(t) d t<\infty \quad \text { a.s. }
$$

(3.10) the positive local martingale $Z_{0}(\cdot)$ is in fact a martingale,

where

$$
\begin{gathered}
\theta(t):=\frac{m(t)-r(t)}{\sigma(t)}-\epsilon \alpha(t) \quad(0 \leq t \leq T), \\
Z_{0}(t):=\exp \left\{-\int_{0}^{t} \theta(s) d B(s)-\frac{1}{2} \int_{0}^{t} \theta^{2}(s) d s\right\} \quad(0 \leq t \leq T) .
\end{gathered}
$$

A sufficient condition for (3.9) and (3.10) is given by the next proposition.

Proposition 3.2. Suppose that there exists a positive constant $c_{1}$ such that

$$
P\left(\frac{|m(t)-r(t)|}{\sigma(t)} \leq c_{1}, \text { Lebesgue-almost-every } t \in[0, T]\right)=1 .
$$

Then (3.9) and (3.10) hold. 
Proof. By Jensen's inequality and the Fubini theorem, we have

$$
E\left[\int_{0}^{T} \alpha(t)^{2} d t\right] \leq \int_{0}^{T} E\left[U(t)^{2}\right] d t=T \gamma(0)<\infty,
$$

where $\gamma(\cdot)$ is the autocovariance function of $U(\cdot)$. Thus (3.13) implies $(3.9)$.

Let $\delta$ be a positive constant such that $\delta<\{2 \gamma(0)\}^{-1}$. By Jensen's inequality, we have, for $t \in[0, T-\delta]$,

$$
\begin{aligned}
\exp \left\{\frac{1}{2} \int_{t}^{t+\delta} \theta^{2}(s) d s\right\} & \leq \frac{\exp \left(\delta c_{1}^{2}\right)}{\delta} \int_{t}^{t+\delta} \exp \left\{\delta \alpha^{2}(s)\right\} d s \\
& \leq \frac{\exp \left(\delta c_{1}^{2}\right)}{\delta} \int_{t}^{t+\delta} E\left[\exp \left\{\delta U^{2}(s)\right\} \mid \mathcal{F}(t)\right] d s
\end{aligned}
$$

Since $U(\cdot)$ is a stationary Gaussian process, we have

$$
E\left[\exp \left\{\delta U^{2}(s)\right\}\right]=\frac{1}{\sqrt{2 \pi \gamma(0)}} \int_{-\infty}^{\infty} \exp \left\{\delta x^{2}\right\} \exp \left\{-\frac{x^{2}}{2 \gamma(0)}\right\} d x<\infty,
$$

whence

$$
E\left[\exp \left\{\frac{1}{2} \int_{t}^{t+\delta} \theta^{2}(s) d s\right\}\right]<\infty \quad(t \in[0, T-\delta]) .
$$

Therefore, by [12, Chapter 3, Corollary 5.14], $Z_{0}(\cdot)$ is a martingale.

Theorem 3.3. We assume (3.9) and (3.10). Then the market $\mathcal{M}$ as defined above is complete. Moreover, if $\sigma(\cdot)$ and $r(\cdot)$ are constant, then, in this market, the prices of European calls and puts are given by the Black-Scholes formulas, and the constant $\sigma$ with $\sigma(\cdot) \equiv \sigma$ serves as the implied volatility.

Proof. The completeness follows from Theorem 3.1 and [13, Theorem 1.6.6]. If $\sigma(\cdot)$ is a positive constant $\sigma$, then from (3.8) we have

$$
S(t) / S_{0}(t)=S(0) \exp \left\{\sigma B_{0}(t)-\frac{\sigma^{2}}{2} t\right\} \quad(0 \leq t \leq T),
$$

where the process $B_{0}(\cdot)$ is defined by

$$
B_{0}(t)=B(t)+\int_{0}^{t} \theta(s) d s \quad(0 \leq t \leq T) .
$$

Since $B_{0}(\cdot)$ is a Brownian motion under the standard martingale measure $P_{0}$ on $\mathcal{F}(T)$ defined by

$$
P_{0}(A):=E\left[Z_{0}(T) 1_{A}\right] \quad(A \in \mathcal{F}(T)),
$$

the behavior of the discounted stock price $S(t) / S_{0}(t)$ under $P_{0}$ is equal to that of the Black-Scholes model. Thus the second half of the theorem follows (cf. [13, Section 2.4]). 
Remark 3.4. In the portfolio optimization problem for standard financial markets, the martingale $Z_{0}(\cdot)$ defined by (3.12) plays an important role (cf. [13]). To explicitly solve the same problem for the financial market $\mathcal{M}$ above, we need to calculate the conditional expectation $\alpha(t)=$ $E[U(t) \mid \mathcal{F}(t)]$ in (3.4) explicitly. This will be done in [3].

\section{Historical volatility}

Let $S(\cdot), U(\cdot)$ and $\gamma(\cdot)$ be as in the previous section. In this section, we assume that $\sigma(\cdot) \equiv \sigma$ with $\sigma$ a positive constant and that $m(\cdot)$ is deterministic. Let $\epsilon$ be as in the previous section. We are concerned with the behavior of the historical volatility $\mathrm{HV}(\cdot)$ defined by (1.7).

Lemma 4.1. Let $t>s \geq 0$. Then

$$
\begin{aligned}
& \operatorname{Var}\{\log (S(t) / S(s))\} \\
& =\left\{(t-s)-2 \epsilon \int_{0}^{t-s} d u \int_{0}^{u} c(v) d v+2 \int_{0}^{t-s} d u \int_{0}^{u} \gamma(v) d v\right\} \sigma^{2} .
\end{aligned}
$$

Proof. We have

$$
\begin{gathered}
\operatorname{Var}\{\log (S(t) / S(s))\}=E\left[\left\{W(t)-W(s)-\epsilon \int_{s}^{t} U(u) d u\right\}^{2}\right] \sigma^{2} \\
=\left\{(t-s)-2 \epsilon E\left[(W(t)-W(s)) \int_{s}^{t} U(u) d u\right]\right. \\
\left.+\int_{s}^{t} \int_{s}^{t} \gamma(u-v) d u d v\right\} \sigma^{2} .
\end{gathered}
$$

By simple calculation, we get $\int_{s}^{t} \int_{s}^{t} \gamma(u-v) d u d v=2 \int_{0}^{t-s} d u \int_{0}^{u} \gamma(v) d v$.

For $s \leq u \leq t$,

$$
E[(W(t)-W(s)) U(u)]=E[(W(u)-W(s)) U(u)]=\int_{s}^{u} c(u-v) d v,
$$

whence

$$
\begin{aligned}
E\left[(W(t)-W(s)) \int_{s}^{t} U(u) d u\right] & =\int_{s}^{t} \int_{s}^{u} c(u-v) d u d v \\
& =\int_{0}^{t-s} d u \int_{0}^{u} c(v) d v
\end{aligned}
$$

Thus the lemma follows.

By Lemma 4.1, we have

$$
\mathrm{HV}(t)=\sigma \sqrt{f(t)} \quad(t>0)
$$


where

$$
f(t):=1-\epsilon \frac{2}{t} \int_{0}^{t} d u \int_{0}^{u} c(v) d v+\frac{2}{t} \int_{0}^{t} d u \int_{0}^{u} \gamma(v) d v \quad(t>0) .
$$

First we consider the model with (1.2) or (2.17).

Theorem 4.2. We assume that $Y(\cdot)$ is as in Theorem 2.13. We also assume that $a(\cdot) \neq 0$, i.e., $\nu \neq 0$. Then $\mathrm{HV}(\cdot)$ is strictly decreasing on $(0, \infty)$, and we have

$$
\begin{gathered}
\operatorname{HV}(t) \uparrow \quad \sigma \quad(t \rightarrow 0+), \\
\operatorname{HV}(t) \downarrow \frac{\sigma}{1+\int_{0}^{\infty} a(u) d u} \quad(t \uparrow \infty) .
\end{gathered}
$$

In particular,

$$
\frac{\sigma}{1+\int_{0}^{\infty} a(u) d u}<\mathrm{HV}(t)<\sigma \quad(0<t<\infty) .
$$

Proof. We have

$$
f^{\prime}(t)=\frac{2}{t}\left\{\frac{1}{t} \int_{0}^{t} d u \int_{0}^{u}(c(v)-\gamma(v)) d v-\int_{0}^{t}(c(v)-\gamma(v)) d v\right\} \quad(t>0) .
$$

Now $c(v)-\gamma(v)>0$ for $v>0$ since

$$
\gamma(v)=\int_{0}^{\infty} c(v+u) c(u) d u<c(v) \int_{0}^{\infty} c(u) d u<c(v)
$$

This implies that $\int_{0}^{t}(c(v)-\gamma(v)) d v$ is strictly increasing in $t$, so that $f^{\prime}(t)<0$ for $t>0$. Thus $f(\cdot)$, hence $\mathrm{HV}(\cdot)$, is strictly decreasing on $(0, \infty)$. From (4.2) with (4.3), we see that $\operatorname{HV}(t) \uparrow \sigma$ as $t \downarrow 0$. Now we have $\int_{0}^{\infty} c(u) d u<1$, and

$$
\int_{0}^{\infty} \gamma(t) d t=\frac{1}{2}\left\{\int_{0}^{\infty} c(u) d u\right\}^{2}
$$

Thus

$$
\lim _{t \rightarrow \infty} f(t)=1-2 \int_{0}^{\infty} c(u) d u+\left(\int_{0}^{\infty} c(u) d u\right)^{2}=\left(1-\int_{0}^{\infty} c(u) d u\right)^{2} .
$$

Since

$$
1-\int_{0}^{\infty} c(u) d u=\left(1+\int_{0}^{\infty} a(u) d u\right)^{-1}
$$

we obtain (4.6) 
Example 4.3. Let $p, q \in(0, \infty)$. Suppose that the stock price $S(\cdot)$ is described by (1.1) with (2.20). Then $c(t)=p e^{-(p+q) t}$ for $t>0$, that is, $S(\cdot)$ is given by

$$
\begin{aligned}
S(t)=S(0) \exp & \left\{\int_{0}^{t} m(s) d s\right. \\
& \left.-\sigma \int_{0}^{t}\left(\int_{-\infty}^{s} p e^{-(p+q)(s-u)} d W(u)\right) d s+\sigma W(t)\right\} .
\end{aligned}
$$

Hence

$$
\gamma(t)=\int_{0}^{\infty} c(t+u) c(u) d u=\frac{p^{2}}{2(p+q)} e^{-(p+q) t} \quad(t>0) .
$$

By Lemma 4.1, we have $\mathrm{HV}(t)=\mathrm{V}(t ; \sigma, p, q)$ for $t>0$ with

$$
\mathrm{V}(t ; \sigma, p, q):=\sigma \sqrt{\frac{q^{2}}{(p+q)^{2}}+\frac{p(2 q+p)}{(p+q)^{3}} \cdot \frac{\left(1-e^{-(p+q) t}\right)}{t}}
$$

and $\operatorname{HV}(t) \downarrow \sigma q /(p+q)$ as $t \uparrow \infty$. In [2], we estimated $\operatorname{HV}(t)(t=$ $1,2,3, \ldots)$ from S\&P 500 closing index. We found that the estimated $\mathrm{HV}(\cdot)$ is not constant, whence the BS model may not be suitable. On the other hand, we fitted the function $\mathrm{V}(t ; \sigma, p, q)$ in (4.8) by using nonlinear least squares, and found that it approximates the estimated $\mathrm{HV}(\cdot)$ reasonably well.

Next we consider the model with (1.3) or (2.10) in the short-memory case.

Theorem 4.4. We assume that $Y(\cdot)$ is as in Theorem 2.11 and that $a(\cdot) \neq 0$. Then $\mathrm{HV}(\cdot)$ is strictly increasing on $(0, \infty)$, and we have

$$
\begin{gathered}
\operatorname{HV}(t) \downarrow \sigma \quad(t \rightarrow 0+), \\
\operatorname{HV}(t) \uparrow \frac{\sigma}{1-\int_{0}^{\infty} a(u) d u} \quad(t \uparrow \infty) .
\end{gathered}
$$

In particular,

$$
\sigma<\operatorname{HV}(t)<\frac{\sigma}{1-\int_{0}^{\infty} a(u) d u} \quad(0<t<\infty) .
$$

The proof of Theorem 4.4 is similar to that of Theorem 4.2; and so we omit it.

Example 4.5. Let $0<p<q$. Suppose that the stock price process $S(\cdot)$ is described by (1.1) with (2.15). Then $c(t)=p e^{-(q-p) t}$ for $t>0$, that is, 
$S(\cdot)$ is given by

$$
\begin{aligned}
S(t)=S(0) & \exp \left\{\int_{0}^{t} m(s) d s\right. \\
& \left.+\sigma \int_{0}^{t}\left(\int_{-\infty}^{s} p e^{-(q-p)(s-u)} d W(u)\right) d s+\sigma W(t)\right\} .
\end{aligned}
$$

Using Lemma 4.1, we have

$$
\mathrm{HV}(t)=\sigma \sqrt{\frac{q^{2}}{(q-p)^{2}}-\frac{p(2 q-p)}{(q-p)^{3}} \cdot \frac{\left(1-e^{-(q-p) t}\right)}{t}}
$$

and $\operatorname{HV}(t) \uparrow \sigma q /(q-p)$ as $t \uparrow \infty$.

Finally we consider the model with (1.3) or (2.10) in the long-memory case.

Theorem 4.6. We assume that $Y(\cdot)$ is as in Theorem 2.7. Then $\mathrm{HV}(\cdot)$ is strictly increasing on $(0, \infty)$, and we have (4.9) and

$$
\mathrm{HV}(t) \uparrow \infty \quad(t \uparrow \infty)
$$

In particular $\mathrm{HV}(t)>\sigma$ for $t>0$. If we further assume (2.12), for $p \in(0,1 / 2)$ and $\ell(\cdot)$ slowly varying at infinity, then

$$
\mathrm{HV}(t) \sim \sigma \frac{t^{p}}{\ell(t)} \frac{\sin (p \pi)}{\pi} \sqrt{\frac{B(1-2 p, p)}{p(2 p+1)}} \quad(t \rightarrow \infty) .
$$

Proof. The first three assertions can be proved in the same way as the proof of Theorem 4.2. So we prove only the last one. By Theorem 2.9, (2.12) implies the following:

$$
\begin{gathered}
c(t) \sim \frac{t^{-(1-p)}}{\ell(t)} \cdot \frac{\sin (p \pi)}{\pi} \quad(t \rightarrow \infty), \\
\gamma(t) \sim \frac{t^{-(1-2 p)}}{\ell(t)^{2}}\left(\frac{\sin (p \pi)}{\pi}\right)^{2} B(1-2 p, p) \quad(t \rightarrow \infty) .
\end{gathered}
$$

We then find that, among the three terms on the right-hand side of (4.3), the first and second terms are negligible relative to the third. Thus we have

$$
f(t) \sim \frac{2}{t} \int_{0}^{t} d u \int_{0}^{u} \gamma(v) d v \sim \frac{t^{2 p}}{\ell(t)^{2}}\left(\frac{\sin (p \pi)}{\pi}\right)^{2} \frac{B(1-2 p, p)}{p(2 p+1)} \quad(t \rightarrow \infty),
$$

which yields (4.15). 
Example 4.7. Let $p \in(0,1 / 2)$. Suppose that $Y(\cdot)$ is as in Theorem 2.7 with $a(\cdot)$ as in Example 2.10. Suppose that $S(\cdot)$ is described by (1.1). Then, by Theorem 4.6, the following holds:

$$
\mathrm{HV}(t) \sim t^{p} \cdot \frac{\sigma \sin (p \pi)}{\pi} \sqrt{\frac{B(1-2 p, p)}{p(2 p+1)}} \quad(t \rightarrow \infty)
$$

\section{Proofs of Theorems 2.1 And 2.3}

We start from a correspondence between $\mu$ and $\nu$ in (2.1) when they are discrete measures.

Lemma 5.1. Let $n \in \mathbf{N}$. Let $\mu$ be a Borel measure on $(0, \infty)$ of the form

$$
\mu=\sum_{k=1}^{n} a_{k} \delta_{r_{k}}
$$

with

$$
\begin{gathered}
a_{k} \in(0, \infty) \quad(k=1,2, \ldots, n), \\
0<r_{1}<r_{2}<\cdots<r_{n}<\infty, \\
\int_{0}^{\infty} \frac{1}{s} \mu(d s)<1 .
\end{gathered}
$$

Then there exists a Borel measure $\nu$ on $(0, \infty)$ of the form

$$
\begin{gathered}
\nu=\sum_{k=1}^{n} b_{k} \delta_{p_{k}} \\
b_{k} \in(0, \infty) \quad(k=1,2, \ldots, n), \\
0<p_{1}<r_{1}<p_{2}<r_{2}<\cdots<p_{n}<r_{n},
\end{gathered}
$$

satisfying (2.1).

Proof. For $w=i z$, we have

$$
\begin{aligned}
\{1 & \left.-\int_{0}^{\infty} \frac{1}{s-w} \mu(d s)\right\}^{-1}-1 \\
& =\left\{\int_{0}^{\infty} \frac{1}{s-w} \mu(d s)\right\}\left\{1-\int_{0}^{\infty} \frac{1}{s-w} \mu(d s)\right\}^{-1} \\
& =\left\{\sum_{k=1}^{n} \frac{a_{k}}{r_{k}-w}\right\}\left\{1-\sum_{k=1}^{n} \frac{a_{k}}{r_{k}-w}\right\}^{-1} \\
& =f(w)^{-1} \sum_{k=1}^{n} a_{k} \prod_{m \neq k}\left(r_{m}-w\right),
\end{aligned}
$$


where $f(w)$ is a polynomial in $w$, of degree $n$, given by

$$
f(w):=\prod_{k=1}^{n}\left(r_{k}-w\right)-\sum_{k=1}^{n} a_{k} \prod_{m \neq k}\left(r_{m}-w\right) .
$$

Now we have

$$
f(0)=\prod_{k=1}^{n} r_{k}-\sum_{k=1}^{n} a_{k} \prod_{m \neq k} r_{m}=\left(\prod_{k=1}^{n} r_{k}\right)\left\{1-\int_{0}^{\infty} \frac{1}{s} \mu(d s)\right\}>0,
$$

and

$$
\operatorname{sgn} f\left(r_{k}\right)=(-1)^{k} \quad(k=1,2, \ldots, n) .
$$

Therefore there exist positive numbers $p_{k}(k=1,2, \ldots, n)$ satisfying $(5.7)$ and

$$
f(w)=\prod_{k=1}^{n}\left(p_{k}-w\right) .
$$

From $f\left(p_{l}\right)=0$, it follows that

$$
\sum_{k=1}^{n} a_{k} \prod_{m \neq k}\left(r_{m}-p_{l}\right)=\prod_{k=1}^{n}\left(r_{k}-p_{l}\right) \quad(l=1,2, \ldots, n) .
$$

So, in the partial fraction decomposition

$$
f(w)^{-1} \sum_{k=1}^{n} a_{k} \prod_{m \neq k}\left(r_{k}-w\right)=\sum_{l=1}^{n} \frac{b_{l}}{p_{l}-w},
$$

the coefficients $b_{l}$ are given by

$$
b_{l}=\frac{\sum_{k=1}^{n} a_{k} \prod_{m \neq k}\left(r_{m}-p_{l}\right)}{\prod_{k \neq l}\left(p_{k}-p_{l}\right)}=\frac{\prod_{k=1}^{n}\left(r_{k}-p_{l}\right)}{\prod_{k \neq l}\left(p_{k}-p_{l}\right)}>0 \quad(l=1,2, \ldots, n) .
$$

With these $p_{l}$ and $b_{l}$, the measure $\nu$ defined by (5.5) gives the desired measure.

Conversely, we have the following lemma.

Lemma 5.2. Let $n \in \mathbf{N}$. Let $\nu$ be a Borel measure on $(0, \infty)$ of the form (5.5) with (5.6) and

$$
0<p_{1}<p_{2}<\cdots<p_{n}<\infty .
$$

Then there exists a Borel measure $\mu$ on $(0, \infty)$ satisfying $(2.1),(5.1)$, (5.2), (5.4), and (5.7).

The proof of Lemma 5.2 is similar to that of Lemma 5.1; and so we omit it. 
We call a Borel measure $\mu$ on $(0, \infty)$ simple if it is of the form $(5.1)$, for some $n \in \mathbf{N}$, with (5.2) and (5.3). We define

$$
\begin{aligned}
\mathcal{M}_{\mathrm{s}} & =\left\{\mu: \begin{array}{l}
\mu \text { is a (possibly zero) simple measure on }(0, \infty) \\
\text { such that } \int_{0}^{\infty} s^{-1} \mu(d s)<1
\end{array}\right\}, \\
\mathcal{N}_{\mathrm{s}} & =\{\nu: \nu \text { is a (possibly zero) simple measure on }(0, \infty)\} .
\end{aligned}
$$

We define the one-to-one and onto map

$$
\theta_{\mathrm{s}}: \mathcal{M}_{\mathrm{s}} \ni \mu \mapsto \nu=\theta_{\mathrm{s}}(\mu) \in \mathcal{N}_{\mathrm{s}}
$$

by $(2.1)$.

In the proofs below, we regard Borel measures $\eta$ on $(0, \infty)$ as Borel measures on $[0, \infty]$ by $\eta\{0\}=\eta\{\infty\}=0$ if necessary.

Proof of Theorem 2.1. We prove only the first half of the theorem; we can prove the second half in a similar fashion. Let $\mu \in \mathcal{M}_{0}$. We define the finite Borel measure $\tilde{\mu}$ on $[0, \infty]$ by

$$
\tilde{\mu}(d s)=s^{-1} I_{(0, \infty)}(s) \mu(d s) .
$$

Take a sequence of simple measures $\mu_{n}(n=1,2, \ldots)$ such that $s^{-1} \mu_{n}(d s)$ converges weakly to $\tilde{\mu}$ on $[0, \infty]$. Since

$$
\tilde{\mu}[0, \infty]=\int_{0}^{\infty} s^{-1} \mu(d s)<1,
$$

we may assume that $\int_{0}^{\infty} s^{-1} \mu_{n}(d s)<1$ for $n=1,2, \ldots$ We put $\nu_{n}:=$ $\theta_{\mathrm{s}}\left(\mu_{n}\right)$ and $\tilde{\nu}_{n}(d s):=s^{-1} \nu_{n}(d s)$. Then we have, for $n=1,2, \ldots$,

$$
\left\{1+\int_{0}^{\infty} \frac{s}{s-i z} \tilde{\nu}_{n}(d s)\right\}\left\{1-\int_{0}^{\infty} \frac{s}{s-i z} \tilde{\mu}_{n}(d s)\right\}=1 \quad(\Im z>0) .
$$

Letting $y \downarrow 0$ in this equality with $z=i y$, we see that

$$
\sup _{n} \tilde{\nu}_{n}[0, \infty]=\sup _{n} \frac{\tilde{\mu}_{n}[0, \infty]}{1-\tilde{\mu}_{n}[0, \infty]}<\infty
$$

Therefore, by the Helly selection principle, we can find a subsequence $n^{\prime}$ such that $\tilde{\nu}_{n^{\prime}}$ converges weakly to $\tilde{\nu}$, say, on $[0, \infty]$. It follows that

$$
\left\{1+\tilde{\nu}\{\infty\}+\int_{0}^{\infty} \frac{1}{s-i z} \nu(d s)\right\}\left\{1-\int_{0}^{\infty} \frac{1}{s-i z} \mu(d s)\right\}=1 \quad(\Im z>0),
$$

where $\nu$ is the measure on $(0, \infty)$ defined by

$$
\nu(d s):=I_{(0, \infty)}(s) s \tilde{\nu}(d s) .
$$

Letting $y \uparrow \infty$ in this equality with $z=i y$, we see that $1+\tilde{\nu}\{\infty\}=1$ or $\tilde{\nu}\{\infty\}=0$. This proves the first half of the theorem. 
Proof of Theorem 2.3. We prove only the first half of the theorem; we can prove the second half in a similar fashion. Let $\mu \in \mathcal{M}_{1}$. Set $m:=$ $\inf \{s: s \in \operatorname{supp}(\mu)\}$. If $m=0$, then

$$
\int_{0}^{\infty} s^{-2} \mu(d s) \leq m^{-1} \int_{[m, \infty)} s^{-1} \mu(d s)<\infty
$$

contradicting the condition $\int_{0}^{\infty} s^{-2} \mu(d s)=\infty$. Thus $m=0$. Therefore there exists an $N \in \mathbf{N}$, such that, for $\mu_{n}(d s):=I_{(1 / n, \infty)}(s) \mu(d s)$,

$$
\int_{0}^{\infty} s^{-1} \mu_{n}(d s)<\int_{0}^{\infty} s^{-1} \mu(d s)=1 \quad(n \geq N),
$$

whence $\mu_{n} \in \mathcal{M}_{0}$ for $n \geq N$. We define $\nu_{n}:=\theta_{0}\left(\mu_{n}\right) \in \mathcal{N}_{0}$. Then, as $n \rightarrow \infty$,

$$
\begin{aligned}
\int_{0}^{\infty} \frac{1}{s+1} \nu_{n}(d s) & =\frac{\int_{0}^{\infty}(1+s)^{-1} \mu_{n}(d s)}{1-\int_{0}^{\infty}(1+s)^{-1} \mu_{n}(d s)} \\
& \rightarrow \frac{\int_{0}^{\infty}(1+s)^{-1} \mu(d s)}{1-\int_{0}^{\infty}(1+s)^{-1} \mu(d s)} \in(0, \infty)
\end{aligned}
$$

so that

$$
\sup _{n} \int_{0}^{\infty} \frac{1}{1+s} \nu_{n}(d s)<\infty
$$

Therefore, for $\tilde{\nu}_{n}(d s):=(s+1)^{-1} I_{(0, \infty)}(s) \nu_{n}(d s)$, there exists a subsequence $n^{\prime}$ such that $\tilde{\nu}_{n^{\prime}}$ converges weakly to a finite Borel measure $\tilde{\nu}$, say, on $[0, \infty]$. It follows that, for $\Im z>0$,

$$
\left\{1-\frac{\tilde{\nu}\{0\}}{i z}+\tilde{\nu}\{\infty\}+\int_{0}^{\infty} \frac{1}{s-i z} \nu(d s)\right\}\left\{1-\int_{0}^{\infty} \frac{1}{s-i z} \mu(d s)\right\}=1,
$$

where $\nu$ is the measure on $(0, \infty)$ defined by $\nu(d s):=(1+s) I_{(0, \infty)}(s) \tilde{\nu}(d s)$.

Letting $y \uparrow \infty$ in the equality above with $z=i y$, we have $\tilde{\nu}\{\infty\}=0$. From $\int_{0}^{\infty} s^{-1} \mu(d s)=1$, it follows that

$$
1-\int_{0}^{\infty} \frac{1}{s+y} \mu(d s)=y \int_{0}^{\infty} \frac{1}{s(s+y)} \mu(d s),
$$

hence

$$
\left\{y+\tilde{\nu}\{0\}+\int_{0}^{\infty} \frac{1}{(s / y)+1} \nu(d s)\right\} \int_{0}^{\infty} \frac{1}{s(s+y)} \mu(d s)=1 \quad(y>0),
$$

and so

$$
\tilde{\nu}\{0\}=\lim _{y \downarrow 0}\left\{\int_{0}^{\infty} \frac{1}{s(s+y)} \mu(d s)\right\}^{-1}=0 .
$$


Finally,

$$
\int_{0}^{\infty} \frac{1}{s} \nu(d s)=\lim _{y \downarrow 0}\left\{\int_{0}^{\infty} \frac{1}{s+y} \mu(d s)\right\}\left\{1-\int_{0}^{\infty} \frac{1}{s+y} \mu(d s)\right\}^{-1}=\infty .
$$

Thus $\nu$ is the desired element of $\mathcal{N}_{1}$.

\section{REFERENCES}

[1] Anh, V. V. And Heyde, C. C. (eds), (1999). Special Issue on Long-Range Dependence. J. Statist. Plann. Inference $\mathbf{8 0 .}$

[2] Anh, V. V., Inoue, A. And Pesee, C. Incorporation of memory into the BlackScholes- Merton theory and estimation of volatility. Submitted.

[3] Anh, V. V., Inoue, A. And Kasahara, Y. Financial markets with memory II: Innovation processes and expected utility maximization. Stochastic Anal. Appl., to appear.

[4] Beran, J. (1994). Statistics of Long-Memory Processes, Chapman \& Hall, New York.

[5] Bingham, N. H., Goldie, C. M. and Teugels, J. L. (1989). Regular Variation, 2nd edn. Cambridge University Press.

[6] Cutland, N. J., Kopp, P. E. And Willinger, W. (1995). Stock price returns and the Joseph effect: a fractional version of the Black-Scholes model. In Seminar on Stochastic Analysis, Random Fields and Applications (Progr. Probab. 36), Birkhäuser, Basel, pp. 327-351.

[7] Comte, F. And Renault, E. (1996). Long memory continuous-time models. $J$. Econometrics 73, 101-149.

[8] Comte, F. and Renault, E. (1998). Long memory in continuous-time stochastic volatility models. Math. Finance 8, 291-323.

[9] Inoue, A. (1993). On the equations of stationary processes with divergent diffusion coefficients. J. Fac. Sci. Univ. Tokyo Sec. IA 40, 307-336.

[10] Inoue, A. (1997). Regularly varying correlation functions and KMO-Langevin equations. Hokkaido Math. J. 26, 1-26.

[11] ITÔ, K. (1954). Stationary random distributions. Mem. Coll. Sci. Univ. Kyoto 28, 209-223.

[12] Karatzas, I. And Shreve, S. E. (1991). Brownian motion and stochastic calculus, 2nd edn. Springer-Verlag, New York.

[13] Karatzas, I. And Shreve, S. E. (1998). Methods of mathematical finance, Springer-Verlag, New York.

[14] Lin, S. J. (1995). Stochastic analysis of fractional Brownian motions. Stochastics Stochastics Rep. 55, 121-140.

[15] Liptser, R. S. And Shiryaev, A. N. (1989). Theory of Martingales, Springer, New York.

[16] Liptser, R. S. And ShiRYAev, A. N. (2001). Statistics of random processes. I. General theory, 2nd edition, Springer, New York.

[17] Rogers, L. C. C. (1997). Arbitrage with fractional Brownian motion. Math. Finance 7, 95-105.

[18] Willinger, W., Taqqu, M. S. and Teverovsky, V. (1999). Stock market prices and long-range dependence. Finance Stoch. 3, 1-13.

E-mail address: v.anh@fsc.qut.edu.au

E-mail address: inoue@math.sci.hokudai.ac.jp 
School of Mathematical Sciences, Queensland University of Technology, GPO Box 2434, Brisbane, Queensland 4001, Australia

Department of Mathematics, Faculty of Science, Hokkaido University, SAPPORO 060-0810, JAPAN 